\title{
Compactness of Fractional Maximal Operator in Weighted and Variable Exponent Spaces
}

\author{
Lütfi AKIN*(iD) \\ Mardin Artuklu University, Faculty of Economics and \\ Administrative Sciences, Department of Business Administration, \\ Mardin, Turkey
}

Geliş / Received: 22/06/2018, Kabul / Accepted: 24/10/2018

\begin{abstract}
We have studied necessary and sufficienty conditions for the weighted fractional maximal operator to be compactness from $L^{p(\cdot)}(0, l)$ to $L^{q(\cdot)}(0, l)$.
\end{abstract}

Keywords: Maximal operator, variable exponent, weight functions

\section{Ağırlıklı ve Değişken Üslü Uzayda Kesirli Maksimal operatörünün Kompaktlığı Öz $L^{p(.)}(0, l)$ 'den $L^{q(.)}(0, l)$ 'e ağırlıklı kesirli maksimal operatörünün kompaktllğı için gerekli ve yeterli şartlar üzerinde çalışıldı.}

Anahtar Kelimeler: Maksimal operator, Değişken üslü, Ağırlık fonksiyonları

\section{Introduction}

In this paper, we will prove compactness of weighted the fractional maximal operator in weighted and variable exponent spaces. Operator theory were worked by very mathematicien (Akin L. 2018; Akin and Zeren, 2017). Recently a considerable number of research has been carried out on the study of generalized Orlicz-Lebesgue spaces $L^{p(x)}$, Orlicz-Sobolev spaces $W^{1, p(x)}$, and the boundedness of different integral operators. In this connection, we refer to the monographs (Cruz-Uribe and Fiorenza, 2013; Diening et al.., 2011; Edmunds et al., 2002; Musielak, 1983; Musielak and Orlicz, 1959). Some necessary and sufficient conditions that assume log-regularity of exponent functions are proved in (Diening and Samko,
2007; Cruz-Uribe and Mamedov, 2012; Mamedov and Harman, 2010; Mamedov and Zeren, 2012; Mamedov and Harman, 2009). The compactness problem of main integral operators in variable exponent Lebesgue spaces are studied in the recent works (Edmunds et al., 2005; Edmunds et al, 1995; Samko,2010; Mamedov et al., 2017). However, excluding the boundedness the compactness problem was little studied for the fractional maximal operator. We note that, there has been no condition of necessary and sufficient character established for the fractional maximal operator yet. We establish compactness of weighted the fractional maximal operator $M_{a}$ necessary and sufficient conditions on measurable almost everywhere positive functions (weights) $v($. 
and $w(),. 0<a<n$, and an open set $\Omega \subset$ $\mathbb{R}^{n}$

$$
\begin{gathered}
M_{a, v, w} f(x)= \\
\sup _{B \ni x} \frac{1}{|B|^{1-a / n}} v(x) \int_{B \cap \Omega} f(y) w(y) d y
\end{gathered}
$$

from $L^{p(x)}(0, l)$ to $L^{q(x)}(0, l)$ with $p(x)$ and $q(x)$ are measurable functions on finite interval $(0, l)$, where the supremum is taken over all balls $B$ which contain $x$. When $a=0$, $M_{0}=M$ is the Hardy-Littlewood maximal operator.

\section{Auxillary Statements and Assertions}

Let $\quad p:(0, l) \rightarrow(1, \infty)$ be a measurable function on finite interval $(0, l)$ We define the space $L^{p(.)}(0, l)$ consisting of all measurable functions $f:(0, l) \rightarrow R$ such that the modular

$$
\rho_{p(.)}(f)=\int_{0}^{l}|f(x)|^{p(x)} d x
$$

is finite.

$$
\begin{aligned}
& \text { If } p^{+}=e s s \sup _{x \in(0, l)} p(x)<\infty, \text { then } \\
& \|f\|_{L^{p(.)}(0, l)}=\inf \left\{\lambda>0: \rho_{p(.)}\left(\frac{f}{\lambda}\right)<\infty\right\}
\end{aligned}
$$

defines a norm on $L^{p(.)}(0, l)$ (Mamedov et al, 2017). Denote by $\chi_{E}$ the characteristic function of the set $E \subset R^{n}$. The weight functions $v$ and $w$ are assumed to be measurable and having non-negative finite values almost everywhere in $(0, l)$ and assume the following summability conditions

$$
\frac{1}{p(x)}+\frac{1}{p^{\prime}(x)}=1
$$

$p^{\prime}$ is conjugate of $p$, $w(x)^{p^{\prime}(x)} \in L^{1}(B(0, a))$, $v(x)^{q(x)} \in L^{1}(a, l)$

For any $a>0$. We use the following notations

$$
V(x)=\int_{x}^{l} v(y)^{q(y)} d y, \quad W(x)=\int_{0}^{x} w(y)^{p^{\prime}(y)} d y .
$$

We use the following general assertions on compact operators.

Theorem 2.1 Mamedov et al (2017); Dunford and Schwartz (1958) let $T \in L(X, Y)$ be a compact operator and $\left(u_{n}\right)$ be a weakly sequence in $E$. The compact $T \in L(X, Y) \quad$ operator every weakly convergent $\left(u_{n}\right)$ sequence converter to strongly convergence sequence.

Theorem 2.2 Rudin (1991) suppose $X, Y$ are Banach space. If $\left\{T_{n}\right\}: X \rightarrow Y$ is a sequence of compact operators in $L(X, Y)$ and $\left\|T_{n}-T\right\|_{X \rightarrow Y} \rightarrow 0$ for some $T \in L(X, Y)$, then $T$ is compact.

We also need for the following corollary.

Corollary 2.3 Okpoti et al (2008) for $s, \alpha, \beta$ positive numbers and $f, g$ measurable functions positive a.e. in $(0, l)$. Denote:

$$
F(x)=\int_{x}^{l} f(t) d t, G(x)=\int_{0}^{x} g(t) d t
$$

and

$B_{1}(x)=F^{\alpha}(x) G^{\beta}(x)$, $B_{4}(x, s)=\left(\int_{0}^{x} f(t) G^{\frac{\beta+s}{\alpha}}(t) d t\right)^{\alpha} G^{-s}(x)$.

Then the numbers

$$
B_{1}=\sup _{0<x<t} B_{1}(x)
$$




$$
B_{4}=\sup _{0<x<t} B_{4}(x)
$$

are mutually equivalent. The constants in the equivalence relation can depend on $s, \alpha, \beta$.

Let $\pi$ be the class of measurable functions $f:(0, l) \rightarrow R$ that have finite values on $(0, l)$ and let $\Lambda_{0}$ be the class of measurable functions $f:(0, l) \rightarrow R$ such that

$$
\lim _{x \rightarrow 0} \sup |f(x)-f(0)| \ln \frac{1}{W(x)}<\infty
$$

Then the following result follows from (Mamedov and Zeren, 2012)

Theorem 2.4 Mamedov and Zeren (2012) let $p, q \in \Lambda_{0} \cap \pi$ and $f(x) \geq 0$ be a measurable functions such that

$$
p^{-}>1, q(0) \geq p(0) \cdot p^{\prime}(0) \text { is }
$$

conjugate of $p(0)$

Then the inequality

$$
\begin{aligned}
& \left\|v(.) H_{v, w} f(.)\right\|_{L^{q^{(.)}(0, l)}} \\
& \leq C\|f(.)\|_{L^{p(.)}(0, l)}
\end{aligned}
$$

holds if and only if

$$
\begin{aligned}
& B=\sup _{0<t<l} B(t) \\
& =\sup _{0<t<l} V(t)^{\frac{1}{q(0)}} \cdot W(t)^{\frac{1}{p^{\prime}(0)}}<\infty
\end{aligned}
$$

Remark 2.5 It is not difficult to see from the proof given in Samko, (2010) that $C \rightarrow O(B)$ as $B \rightarrow 0$. See inequalities (2), $C$ is constant and see (Diening et al, 2011) for $B$.

We also need for the following lemma..

Lemma 2.6 Mamedov et al (2017). Let the function $W(x)$ be as above. $c$ is a constant. Then it follows that

$$
W(t)^{-\frac{1}{p(s)}} \geq \frac{1}{c} \cdot W(t)^{-\frac{1}{p(x)}}
$$

for $0<s<x<t$.

Lemma 2.7 Mamedov et al (2017). For the function $W(x)$ and constant $c$ it follows that

$W(t)^{-\frac{q(x)}{p(x)}} \geq \frac{1}{c} \cdot W(t)^{-\frac{q(0)}{p(0)}}$,

for $0<x<t<l$.

Lemma 2.8 Mamedov et al (2017). For the function $W(x)$ and constant $c$ it follows that $W(x)^{q(x)} \geq \frac{1}{c} . W(x)^{q(0)}$.

\section{Main Result}

Theorem 3.1. Let $p, q \in \Lambda_{0} \cap \pi \quad, B=$ $\sup _{0<x<l} B(x)$ and $f(x) \geq 0$ be a measurable functions such that $p^{-}>1$,

$$
\left(p^{-}=\text {ess } \inf _{x \in(0, l)} p(x)\right)
$$

$q(0) \geq p(0)>1 . p^{\prime}$ is conjugate of $p$. Then $M_{a, v, w} f(x)$ is compact from $L^{p(.)}(0, l)$ to $L^{q(.)}(0, l)$ if and only if

$$
\begin{aligned}
& \limsup _{t \rightarrow 0} V(t)^{1 / q(0)} W(t)^{1 / p^{\prime}(0)}=0 \\
& \sup _{t \in(0, \infty)} V(t)^{1 / q(0)} W(t)^{1 / p^{\prime}(0)}<\infty
\end{aligned}
$$
and

Proof. Sufficiency. Following Cruz-Uribe and Mamedov (2012), set

$K_{1} f(x)=$

$$
\chi_{(0, \delta)}(x) \sup _{B \ni x} \frac{1}{|B|^{1-a / n}} v(x) \int_{0}^{x} f(t) w(t) d t .
$$

$K_{2} f(x)=$ 


$$
\begin{aligned}
& \chi_{(\delta, 1)}(x) \sup _{B \ni x} \frac{1}{|B|^{1-a / n}} v(x) \int_{0}^{\delta} f(t) w(t) d t . \quad f_{t}(x)=\left(\int_{0}^{t} w(\theta)^{p^{\prime}(\theta)} d \theta\right)^{-\frac{1}{p(s)}} . \\
& \cdot \chi_{(0, t)}(x) w(x)^{p^{\prime}(x)-1}
\end{aligned}
$$$$
K_{3} f(x)=
$$$$
\chi_{(\delta, 1)}(x) \sup _{B \ni x} \frac{1}{|B|^{1-a / n}} v(x) \int_{\delta}^{x} f(t) w(t) d t . \quad \text { It follows that }
$$

into the inequality (2).

$a \in(0,1)$

and

$B(t)=V(t)^{\frac{1}{q(0)}} W(t)^{\frac{1}{p^{\prime}(0)}}$.

Then

$M_{a, v, w} f(x)=\sum_{i=1}^{3} K_{i} f(x)$

$=K_{1} f(x)+K_{2} f(x)+K_{3} f(x)$

Taking into account Lemma 2 from Edmunds et al. (1994) we find that $K_{3}$ is a norm limit of a sequence of finite rank operators, while $K_{2}$ is a finite rank operator. Now using Theorem 2.4, Remark 2.5 and the condition $\lim _{t \rightarrow 0} B(t)=0$ it follows that

$\left\|K_{1} f(x)\right\|_{q(.),(0, l)}$

$=\left\|\sup _{B \ni x} \frac{1}{|B|^{1-a / n}} v(x) \int_{0}^{x} f(t) w(t) d t\right\|_{q(.),(0, l)}$

$\leq C\|f\|_{q(.),(0, l)}$

Hence

$$
\begin{gathered}
\left\|M_{a, v, w} f-K_{2}-K_{3}\right\|_{L^{p(.) \rightarrow L^{q(.)}}} \\
=\left\|K_{1}\right\|_{L^{p(.) \rightarrow L^{q(.)}}} \\
=O\left(\min _{0<t<\delta} B(t)\right) \rightarrow 0
\end{gathered}
$$

as $t \rightarrow 0$.

This completes the proof of the sufficiency part of Theorem 3.1.

Necessity. Let the test function

$$
\rho_{p}\left(f_{t}\right)=
$$

$$
\begin{aligned}
& \int_{0}^{1}\left(\begin{array}{l}
\left(\int_{0}^{t} w(\theta)^{p^{\prime}(\theta)} d \theta\right)^{-\frac{1}{p(s)}} \\
\cdot \chi_{(0, t)}(x) w(x)^{p^{\prime}(x)-1}
\end{array}\right) d x \\
& \leq\left(\int_{0}^{t} w(x)^{p^{\prime}(x)} d x\right)\left(\int_{0}^{t} w(\theta)^{p^{\prime}(\theta)} d \theta\right)^{-1} \\
& \leq 1 .
\end{aligned}
$$

Therefore, $\quad \rho_{p}\left(f_{t}\right) \leq 1$. From the elementary properties of the variable exponent norms it follows that Cruz-Uribe and Fiorenza (2013); Diening et al. (2011)

$$
\left\|f_{t}(x)\right\|_{L^{p(\cdot)}(0,1)} \leq 1 \text {. }
$$

Therefore, by Hölder's inequality we have

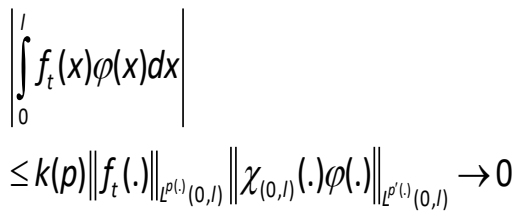

as $t \rightarrow 0$ for all $\varphi \in L^{p^{\prime}(.)}(0, l)$. Hence $f_{t}$ converges weakly to 0 . Hence, by compactness hypothesis of the operator $M_{a, v, w} f$, and Theorem 2.1 it follows that the sequence $\left\{M_{a, v, w} f_{t}\right\}$ converges to 0 in the norm of $L^{q(.)}(0, I)$. Therefore, 
$\rho_{p}\left(M_{a, v, w} f_{t}\right) \rightarrow 0$ as $t \rightarrow 0$

On the other hand,

$$
\begin{gathered}
\rho_{p}\left(M_{a, v, w} f_{t}\right)= \\
\int_{0}^{1}\left(\begin{array}{c}
\sup _{B \ni x} \frac{1}{|B|^{(1-a / n) / q(x)}} v(x) \\
\cdot \int_{0}^{x} \quad\left(\int_{0}^{t} w(\theta)^{p^{\prime}(\theta)} d \theta\right)^{-\frac{1}{p(s)}} \\
\cdot \chi_{(0, t)}(s) w(s)^{p^{\prime}(s)-1} w(s) d s
\end{array}\right) \\
\quad \int_{0}^{q(x)} \sup _{B \ni x} \frac{1}{|B|^{1-a / n}} v(x)^{q(x)} \\
\left(\int_{0}^{x}\left(\int_{0}^{t} w(\theta)^{p^{\prime}(\theta)} d \theta\right)^{-\frac{1}{p(s)}}\right)^{q(x)} d x(6) \\
\cdot w(s)^{p^{\prime}(s)} d s
\end{gathered}
$$

(by Lemma 2.6),

$$
\geq(2 C)^{-q^{+}} \int_{0}^{t} V(x)^{q(x)} W(x)^{q(x)} W(t)^{-\frac{q(x)}{p(x)}} d x
$$

(by Lemma 2.7),

$$
\geq \frac{2^{-q^{+}}}{C_{1}} W(t)^{-\frac{q(0)}{p(0)}} \int_{0}^{t} V(x)^{q(x)} W(x)^{q(x)} d x
$$

(by Lemma 2.8),

$$
\geq \frac{1}{C_{2}}\left[W(t)^{-\frac{1}{p(0)}}\left(\int_{0}^{t} V(x)^{q(x)} W(x)^{q(0)} d x\right)^{\frac{1}{q(0)}}\right]^{q(0)}
$$

(by Corollary 2.3 ),

$$
\geq C_{3}\left[V(t)^{\frac{1}{q(0)}} W(t)^{\frac{1}{p^{\prime}(0)}}\right]^{q(0)}
$$

from this inequality and (5) it follows that
$V(t)^{\frac{1}{q(0)}} W(t)^{\frac{1}{p^{(}(0)}} \rightarrow 0$ as $t \rightarrow 0$

The necessity of Theorem 3.1 has been proved.

Notice, in the proof of inequality (6), we have applied Corollary 2.3 under the settings:

$$
\begin{aligned}
d x \quad F(t) & =V(t)=\int_{t}^{1} v(x)^{q(x)} d x, \\
G(t) & =W(t)=\int_{0}^{t} w(x)^{p^{\prime}(x)} d x
\end{aligned}
$$

and

$$
\alpha=\frac{1}{q(0)}, \beta=\frac{1}{p^{\prime}(0)}, s=\frac{1}{p(0)}, f(t)=v(x)^{q(x)}, g(t)=w(x)^{p^{\prime}(x)} .
$$

Then

$\left(\int_{0}^{t} f(x) G(x)^{\frac{\beta+s}{\alpha}} d x\right)^{\alpha} G(t)^{-s}=\left(\int_{0}^{t} v(x)^{q(x)} W(x)^{\alpha(0)} d x\right)^{\frac{1}{q(0)}} W(t)^{-\frac{1}{\rho(0)}}$

(by Corollary 2.3),

$\geq C F(t)^{\alpha} G(t)^{\beta}=C V(t)^{\frac{1}{q(0)}} W(t)^{\frac{1}{p^{\prime}(0)}}$

This completes the proof of Theorem 3.1.

Conclusion. We have shown the necessary and sufficient conditions for the compactness of thefractional maximal operator in the weighted and variable exponential space.

Acknowledgements. The author would like to thanks the reviewer for his/her valuable suggestions that enhance the presentation of this paper.

\section{References}

Akin L.2018, "A Characterization of Approximation of Hardy Operators in $V L S$ ",Celal Bayar University Journal of Science, Volume 14, Issue 3, pp:333-336.

Akin L. and Zeren Y. 2017, "Approximation to generalized Taylor derivatives by integral 
operator families”, MSU J. Sci. Volume 5, Issue 2, p 421-423.

Cruz-Uribe D. and Fiorenza A. 2013, "Variable Lebesgue Spaces. Foundations and Harmonic Analysis". Birkhauser.

Cruz.-Uribe D. and Mamedov F.I. 2012, “On a general weighted Hardy type inequality in the variable exponent Lebesgue spaces," Rev. Mat. Compl., 25(2), 335-367.

Diening L. and Samko S. 2007, "Hardy inequality in variable exponent Lebesgue spaces," Frac. Calc. \& Appl. Anal., 10(1), 117.

Diening L., Harjulehto P., Hasto P. and Ruzicka W. 2011, "Lebesgue and Sobolev Spaces with Variable Exponents," Lecture Not. in Math., Springer, Heidelberg, Germany

Dunford N. and Schwartz J.T. 1958, "Linear operators: general theory, Part 1," WileyInterscience, New York.

Edmunds D.E., Kokilashvili V. and Meskhi A. 2002, "Bounded and compact integral operators" Mathematics and its Applications, 543. Kluwer Academic Publishers, Dordrecht.

Edmunds D.E., Kokilashvili V. and Meskhi A.2005, "On the boundedness and compactness of the weighted Hardy operators in spaces," Georgian Math. J., 12(1), 27-44.

Edmunds D.E., Gurka P. and Pick L.1994, "Compactness of Hardy-type integral operators in weighted Banach function spaces”. Studia Math. 109(1),73-90.

Musielak J. 1983, “Orlicz spaces and modular spaces". Lecture Note in Mathematics, 1034. Springer-Verlag, Berlin.

Musielak J. and Orlicz W. 1959, "On modular spaces." Studia Math.18, 49-65.

Mamedov F.I. and Harman A. 2010, "On a Hardy type general weighted inequality in spaces $\operatorname{Lp}($.$) , ” Integral Eq. Oper. Theory, 66$ (4), 565-592

Mamedov F.I. and Zeren Y. 2012, "On equivalent conditions for the general weighted Hardy type inequality in space Lp..)," Zeitsch. Anal. Anw., 31 (1), 55-74.

Mamedov F.I. and Harman A. 2009, "On a weighted inequality of Hardy type in spaces Lp(.)," J. Math. Anal. Appl., 353 (2), 521530.

Mamedov F.I., Zeren Y. and Akin L. 2017, "Compactification of weighted Hardy operator in variable exponent Lebesgue spaces", Asian Journal of Mathematics and Computer Research, 17(1): 38-47.

Okpoti C.A., Persson L.E. and Sinnamon O. 2008, "An equivalence theorem for some integral conditions with general measures related to Hardy's inequality II." Journal Math. Anal. Appl. 337(1),219230.

Rudin W. 1991, "FunctionalAnalysis", McGraw-Hill, Inc. Singapor.

Samko S. 2010, "On compactness of operators in variable exponent Lebesgue spaces", Operator Theory: Adv. and Appl., 202, 497-508. 